\title{
An Efficient Synthesis of 2,4,5-Trisubstituted and 1,2,4,5-Tetrasubstituted-1H-imidazoles
}

\author{
Mazaahir Kidwai, "Shilpi Saxena, Ruby, and Shweta Rastogi \\ Department of Chemistry, University of Delhi, Delhi-110007, India. *E-mail: kidwai_chemistry@yahoo.co.uk \\ Received July 26, 2005
}

Key Words : Microwave irradiation, Neat reaction, 1,2,4,5-Tetrasubstituted imidazole, 2,4,5-Trisubstituted imidazole, Green chemistry

Since the appearance of the first paper on the application of microwave irradiation in the chemical synthesis ${ }^{1}$ the approach has blossomed into a useful technique for a variety of applications in organic synthesis ${ }^{2}$ and functional group transformations. ${ }^{3}$ In moving towards sustainable technologies the focus has now shifted to less cumbersome solvent-

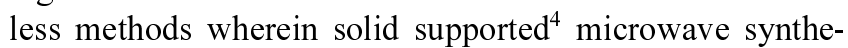
$\mathrm{sis}^{5,6}$ has played an important role. This technique eliminates the use of solvent during the reaction stage but requires an appreciable amount of solvent for the adsorption of reagents and elution of product. The emergence of neat reaction is a recent approach under the new paradigm of green chemistry ${ }^{7}$ where the neat reactants undergo facile reaction to provide high yield of pure products thus eliminating or minimizing the use of organic solvents. These no solvent ${ }^{8}$ reactions are not only advantageous for environmental reasons but also offer benefits of enhanced reaction rates, greater selectivity and experimental ease of manipulation.

The imidazole ring system is of particular interest as it is a component of histidine that produces histamine in metabolic process. $^{9}$ The potency and wide applicability of the imidazole pharmacophore can be attributed to its hydrogen bond donor-acceptor capability as well as its high affinity for metals which are present in many protein active sites (eg. $\mathrm{Zn}, \mathrm{Fe}, \mathrm{Mg}){ }^{10}$ Triaryl imidazoles are used in photography ${ }^{11}$ as photosensitive compounds. In addition, they are of interest because of their herbicidal, ${ }^{12}$ analgesic, ${ }^{13}$ fungicidal, ${ }^{14}$ antiinflammatory ${ }^{15}$ and antithrombotic activities. ${ }^{16}$

The original synthesis of imidazole utilized glyoxal, formaldehyde and ammonia and established that the formation of four N-C bonds was a viable route. ${ }^{17,18}$ Although classical methods were derived from this early success, the reactions suffered low yields, mixture of products and lack of generality. Synthetic methodology alternatives are many ${ }^{13}$ and varied ${ }^{19,20}$ and have resorted to harsh conditions (eg. the formamide synthesis, which requires excess reagents, $\mathrm{H}_{2} \mathrm{SO}_{4}$ as a condensing agent, $\left.150-200{ }^{\circ} \mathrm{C}, 4-6 \mathrm{~h}, 40-90 \%\right) .{ }^{21,22}$ Also, reagents for these procedures are not readily available, a key deficiency for library synthesis.

In continuation to our endeavour towards green chemistry $\mathrm{y}^{23}$ and the biological importance of substituted imidazole derivatives we have developed an ecologically safe strategy for the synthesis of 2,4,5-trisubstituted and 1,2,4,5-tetrasubstituted imidazole derivatives employing neat reaction conditions using MWI.

\section{Results and Discussion}

The synthesis of tri/tetra substituted imidazoles by the condensation of aldehyde, benzil, ammonium acetate and amine in refluxing acetic acid for a few hours is a well established procedure. ${ }^{24}$ However, this method suffers from drawbacks which include drastic reaction conditions, longer reaction time with tedious work up and low yield of products. In contrast to the drawbacks of classical procedure an improved methodology for the synthesis of 2,4,5trisubstituted and 1,2,3,4-tetrasubstituted imidazoles using zeolite, silica gel and acidic alumina under MWI is reported. ${ }^{19}$ But this technique does not exactly meet the definition of 'no solvent' as it requires the use of an appreciable amount of solvent at the pre and post reaction stages.

We have modified the solid supported technique to an environmentally friendly neat synthesis ${ }^{25}$ in which the reaction is carried out in the absence of solvent, solid support and acid. Equimolar amount of neat reactants viz. benzil, aldehydes 1a-h and excess of ammonium acetate on exposure to microwave irradiation gave excellent yield (Table 1) of 2,4,5-trisubstituted $1 \mathrm{H}$ imidazoles 2a-h (Scheme 1) after

Table 1. Reaction Times and Yields for Compounds 2a-h and 4a-h

\begin{tabular}{cccccc}
\hline $\begin{array}{c}\text { Compd. } \\
\text { No. }\end{array}$ & $\mathrm{R}^{1}$ & $\mathrm{R}^{2}$ & $\mathrm{mp}^{\circ}{ }^{\circ} \mathrm{C}$ & $\begin{array}{c}\text { Time } \\
(\mathrm{min} .)\end{array}$ & $\begin{array}{c}\text { Yield } \\
(\%)\end{array}$ \\
\hline $\mathbf{2 a}$ & Phenyl & - & $276-277^{26 \mathrm{a}}$ & 1.5 & 96 \\
$\mathbf{2 b}$ & 4-Cl-Phenyl & - & $263-264^{26 \mathrm{~b}}$ & 1.8 & 94 \\
$\mathbf{2 c}$ & Piperonyl & - & $201-202$ & 2.1 & 93 \\
$\mathbf{2 d}$ & 2-chloro-3-quinolyl & - & $137-138$ & 2.8 & 90 \\
$\mathbf{2 e}$ & 4-OH-Phenyl & - & $256-257^{27 \mathrm{a}}$ & 2.5 & 91 \\
$\mathbf{2 f}$ & Indolyl & - & $298-299$ & 3.1 & 92 \\
$\mathbf{2 g}$ & 4-N(CH$)_{2} \mathrm{C}_{6} \mathrm{H}_{4}$ & - & $259-260^{26 b}$ & 2.6 & 95 \\
$\mathbf{2 h}$ & Furyl & - & $235-237$ & 2.3 & 90 \\
$\mathbf{4 a}$ & Phenyl & Phenyl & $217-218^{28}$ & 2.1 & 93 \\
$\mathbf{4 b}$ & Phenyl & Benzyl & $160-162^{27}$ & 2.9 & 91 \\
$\mathbf{4 c}$ & 4-Cl-Phenyl & Phenyl & $158-160$ & 2.7 & 95 \\
$\mathbf{4 d}$ & 4-Cl-Phenyl & Benzyl & $164-165^{13}$ & 3.0 & 90 \\
$\mathbf{4 e}$ & Piperonyl & Phenyl & $198-199$ & 2.5 & 95 \\
$\mathbf{4 f}$ & Piperonyl & Benzyl & $148-150^{13}$ & 2.9 & 94 \\
$\mathbf{4 g}$ & 2-chloro-3-quinolyl & Phenyl & $107-108$ & 2.1 & 89 \\
$\mathbf{4 h}$ & 2-chloro-3-quinolyl & Benzyl & $94-95$ & 2.0 & 88 \\
\hline
\end{tabular}

Microwave heating $\left(800 \mathrm{~W}, 2450 \mathrm{MHz}, 110-120^{\circ} \mathrm{C}, 1 \mathrm{~min}\right)$ 
<smiles>[R]c1nc(-c2ccccc2)c(-c2ccccc2)[nH]1</smiles>

2a-h

Scheme 1

triturating with few drops of methanol. Inspired by the positive results obtained for the synthesis of 2,4,5-trisubstituted imidazoles similar technique was applied for the

synthesis of 1,2,4,5-tetrasubstituted $1 \mathrm{H}$ imidazoles 4a-h which were also obtained in high yields within few minutes of MWI by the four component condensation of benzil, aldehyde 1a-d, aromatic amine 3a-b and ammonium acetate (Scheme 2). The neat reaction was also attempted under conventional heating, keeping similar reaction conditions. Direct heating of reactants without solvent took more time for completion of reaction and gave the products in low yields and in most cases, lead to charring. The structure of the synthesized compounds was confirmed by spectroscopic and analytical data (Table 2). In the IR spectra the absence of the carbonyl and aldehyde bands are in the accordance with

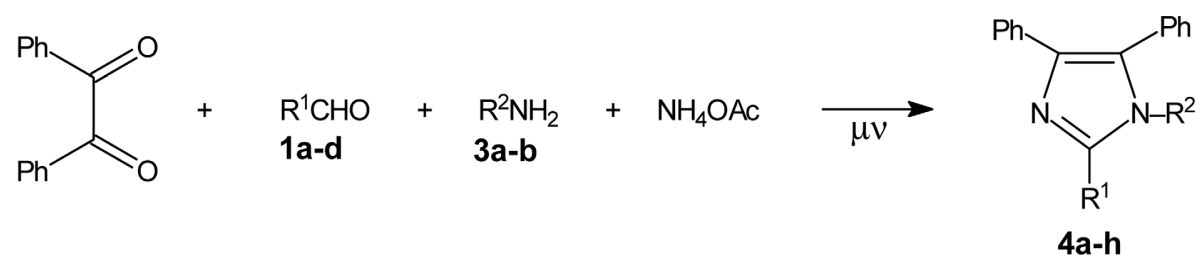

Scheme 2. $\mathrm{R}^{1}=\mathrm{a}=$ phenyl, $\mathrm{b}=4$-Cl-phenyl, $\mathrm{c}=$ piperonyl, $\mathrm{d}=$ 2-chloro-3-quinolyl, $\mathrm{e}=4$-OH-phenyl, $\mathrm{f}=$ indolyl, $\mathrm{g}=4-\mathrm{N}\left(\mathrm{CH}_{3}\right)_{2}$-phenyl, $\mathrm{h}=$ furyl. $\mathrm{R}^{2}=\mathrm{a}=$ phenyl, $\mathrm{b}=$ benzyl

Table 2. Analytical and Spectral Data for Compounds 2a-h and $\mathbf{4 a - h}$

\begin{tabular}{|c|c|c|c|c|c|c|}
\hline \multirow{2}{*}{$\begin{array}{c}\text { Compd. } \\
\text { No. }\end{array}$} & \multirow{2}{*}{$\begin{array}{l}\text { Molecular } \\
\text { Formula }\end{array}$} & \multicolumn{3}{|c|}{$\%$ CHN, Found (Calcd) } & \multirow{2}{*}{ IR (in $\mathrm{KBr}$ ) $\mathrm{cm}^{-1}$} & \multirow{2}{*}{${ }^{1} \mathrm{H} \mathrm{NMR}\left(\mathrm{CDCl}_{3}+\mathrm{DMSO}, \delta, 300 \mathrm{MHz}\right)$} \\
\hline & & $\mathrm{C}$ & $\mathrm{H}$ & $\mathrm{N}$ & & \\
\hline $2 \mathbf{a}$ & $\mathrm{C}_{21} \mathrm{H}_{16} \mathrm{~N}_{2}$ & $\begin{array}{c}85.18 \\
(85.13)\end{array}$ & $\begin{array}{c}5.33 \\
(5.40)\end{array}$ & $\begin{array}{c}9.38 \\
(9.45)\end{array}$ & $\begin{array}{l}1600(\mathrm{C}=\mathrm{C}), 1580(\mathrm{C}=\mathrm{N}) \\
3445(\mathrm{~N}-\mathrm{H})\end{array}$ & 7.15-7.92 (m, 15H, Ar-H), 9.20 (brs, NH) \\
\hline $2 \mathbf{b}$ & $\mathrm{C}_{21} \mathrm{H}_{15} \mathrm{ClN}_{2}$ & $\begin{array}{c}76.18 \\
(76.24)\end{array}$ & $\begin{array}{c}4.58 \\
(4.53)\end{array}$ & $\begin{array}{c}8.40 \\
(8.47)\end{array}$ & $\begin{array}{l}1615(\mathrm{C}=\mathrm{C}), 1584(\mathrm{C}=\mathrm{N}) \\
3452(\mathrm{~N}-\mathrm{H})\end{array}$ & $\begin{array}{l}7.10-7.60(\mathrm{~m}, 10 \mathrm{H}, \mathrm{Ar}-\mathrm{H}), 7.35(\mathrm{~d}, 2 \mathrm{H}, \mathrm{Ar}), 7.85(\mathrm{~d}, \\
2 \mathrm{H}, \mathrm{Ar}), 9.30 \text { (brs, NH) }\end{array}$ \\
\hline $2 \mathrm{c}$ & $\mathrm{C}_{22} \mathrm{H}_{16} \mathrm{~N}_{2} \mathrm{O}_{2}$ & $\begin{array}{c}77.58 \\
(77.64)\end{array}$ & $\begin{array}{c}4.76 \\
(4.70)\end{array}$ & $\begin{array}{c}8.18 \\
(8.23)\end{array}$ & $\begin{array}{l}1607(\mathrm{C}=\mathrm{C}), 1589(\mathrm{C}=\mathrm{N}) \\
3440(\mathrm{~N}-\mathrm{H})\end{array}$ & $\begin{array}{l}5.90\left(\mathrm{~s}, 2 \mathrm{H}, \mathrm{OCH}_{2} \mathrm{O}\right), 6.71-7.20(\mathrm{~m}, 3 \mathrm{H}, \text { piperonal), } \\
7.32-7.82(\mathrm{~m}, 10 \mathrm{H}, \mathrm{Ar}-\mathrm{H}), 9.41 \text { (brs, NH) }\end{array}$ \\
\hline 2d & $\mathrm{C}_{24} \mathrm{H}_{16} \mathrm{ClN}_{3}$ & $\begin{array}{c}75.43 \\
(75.49)\end{array}$ & $\begin{array}{c}4.12 \\
(4.19)\end{array}$ & $\begin{array}{c}11.08 \\
(11.00)\end{array}$ & $\begin{array}{l}1625(\mathrm{C}=\mathrm{C}), 1578(\mathrm{C}=\mathrm{N}) \\
3435(\mathrm{~N}-\mathrm{H})\end{array}$ & $\begin{array}{l}\text { 7.21-8.35 (m, 15H, Ar-H + Quinolyl), } 9.32 \text { (brs, } \\
\mathrm{NH})\end{array}$ \\
\hline $2 \mathbf{e}$ & $\mathrm{C}_{21} \mathrm{H}_{16} \mathrm{~N}_{2} \mathrm{O}$ & $\begin{array}{c}80.82 \\
(80.76)\end{array}$ & $\begin{array}{c}5.15 \\
(5.12)\end{array}$ & $\begin{array}{c}8.92 \\
(8.97)\end{array}$ & $\begin{array}{l}1615(\mathrm{C}=\mathrm{C}), 1582(\mathrm{C}=\mathrm{N}) \\
3200(\mathrm{OH}), 3450(\mathrm{~N}-\mathrm{H})\end{array}$ & 6.70-7.61 (m, 15H, Ar-H), 9.52 (brs, NH) \\
\hline $2 f$ & $\mathrm{C}_{23} \mathrm{H}_{17} \mathrm{~N}_{3}$ & $\begin{array}{c}82.20 \\
(82.14)\end{array}$ & $\begin{array}{c}5.28 \\
(5.35)\end{array}$ & $\begin{array}{c}12.42 \\
(12.50)\end{array}$ & $\begin{array}{l}1628(\mathrm{C}=\mathrm{C}), 1588(\mathrm{C}=\mathrm{N}) \\
3452(\mathrm{NH})\end{array}$ & $\begin{array}{l}\text { 7.12-7.91 (m, 15H, Ar-H), } 9.20 \text { (brs, N-H), } 10.21 \\
(\mathrm{~s}, 1 \mathrm{H}, \mathrm{NH} \text { indole) }\end{array}$ \\
\hline $2 \mathrm{~g}$ & $\mathrm{C}_{23} \mathrm{H}_{21} \mathrm{~N}_{3}$ & $\begin{array}{c}81.47 \\
(81.41)\end{array}$ & $\begin{array}{c}6.12 \\
(6.19)\end{array}$ & $\begin{array}{c}12.30 \\
(12.38)\end{array}$ & $\begin{array}{l}1620(\mathrm{C}=\mathrm{C}), 1598(\mathrm{C}=\mathrm{N}) \\
3415(\mathrm{~N}-\mathrm{H})\end{array}$ & $\begin{array}{l}2.90\left(\mathrm{~s}, 2 \mathrm{CH}_{3}\right), 6.60(\mathrm{~d}, 2 \mathrm{H}, \mathrm{Ar}-\mathrm{H}), 7.10-7.62(\mathrm{~m}, \\
10 \mathrm{H}, \mathrm{Ar}-\mathrm{H}), 7.70(\mathrm{~d}, 2 \mathrm{H}, \mathrm{Ar}-\mathrm{H}), 9.12(\mathrm{brs}, \mathrm{NH})\end{array}$ \\
\hline $2 \mathrm{~h}$ & $\mathrm{C}_{19} \mathrm{H}_{14} \mathrm{~N}_{2} \mathrm{O}_{2}$ & $\begin{array}{c}75.42 \\
(75.49)\end{array}$ & $\begin{array}{c}4.58 \\
(4.63)\end{array}$ & $\begin{array}{c}9.21 \\
(9.27)\end{array}$ & $\begin{array}{l}1624(\mathrm{C}=\mathrm{C}), 1594(\mathrm{C}=\mathrm{N}) \\
3430(\mathrm{~N}-\mathrm{H})\end{array}$ & $\begin{array}{l}\text { 6.12-6.40 (m, 2H, furan), 7.20-7.92 (m, 11H, Ar-H } \\
+ \text { furan), } 9.30 \text { (brs, NH) }\end{array}$ \\
\hline $4 \mathbf{a}$ & $\mathrm{C}_{27} \mathrm{C}_{20} \mathrm{~N}_{2}$ & $\begin{array}{c}87.15 \\
(87.09)\end{array}$ & $\begin{array}{c}5.32 \\
(5.37)\end{array}$ & $\begin{array}{c}7.58 \\
(7.52)\end{array}$ & $1625(\mathrm{C}=\mathrm{C}), 1596(\mathrm{C}=\mathrm{N})$ & 7.03-7.61 (m, 20H, Ar-H) \\
\hline $4 \mathrm{~b}$ & $\mathrm{C}_{28} \mathrm{H}_{22} \mathrm{~N}_{2}$ & $\begin{array}{c}87.10 \\
(87.04)\end{array}$ & $\begin{array}{c}5.62 \\
(5.69)\end{array}$ & $\begin{array}{c}7.28 \\
(7.25)\end{array}$ & $1621(\mathrm{C}=\mathrm{C}), 1589(\mathrm{C}=\mathrm{N})$ & $5.16\left(\mathrm{~s}, 2 \mathrm{H},-\mathrm{CH}_{2}-\right), 6.68-7.71(\mathrm{~m}, 20 \mathrm{H}, \mathrm{Ar}-\mathrm{H})$ \\
\hline $4 c$ & $\mathrm{C}_{27} \mathrm{H}_{19} \mathrm{ClN}_{2}$ & $\begin{array}{c}79.76 \\
(79.70)\end{array}$ & $\begin{array}{c}4.62 \\
(4.67)\end{array}$ & $\begin{array}{c}6.82 \\
(6.88)\end{array}$ & $1605(\mathrm{C}=\mathrm{C}), 1594(\mathrm{C}=\mathrm{N})$ & $\begin{array}{l}\text { 7.10-7.60 (m, 15H, Ar-H), } 7.32(\mathrm{~d}, 2 \mathrm{H}, \mathrm{Ar}), 7.80(\mathrm{~d} \text {, } \\
2 \mathrm{H}, \mathrm{Ar})\end{array}$ \\
\hline 4d & $\mathrm{C}_{28} \mathrm{H}_{21} \mathrm{ClN}_{2}$ & $\begin{array}{c}79.96 \\
(79.90)\end{array}$ & $\begin{array}{c}4.92 \\
(4.99)\end{array}$ & $\begin{array}{c}6.88 \\
(6.65)\end{array}$ & $1615(\mathrm{C}=\mathrm{C}), 1598(\mathrm{C}=\mathrm{N})$ & $\begin{array}{l}5.15\left(\mathrm{~s}, 2 \mathrm{H},-\mathrm{CH}_{2}-\right), 7.10-7.60(\mathrm{~m}, 15 \mathrm{H}, \mathrm{Ar}-\mathrm{H}), 7.35 \\
(\mathrm{~d}, 2 \mathrm{H}, \mathrm{Ar}), 7.85(\mathrm{~d}, 2 \mathrm{H}, \mathrm{Ar})\end{array}$ \\
\hline $4 e$ & $\mathrm{C}_{28} \mathrm{H}_{20} \mathrm{~N}_{2} \mathrm{O}_{2}$ & $\begin{array}{c}80.71 \\
(80.76)\end{array}$ & $\begin{array}{c}4.86 \\
(4.80)\end{array}$ & $\begin{array}{c}6.78 \\
(6.73)\end{array}$ & $1619(\mathrm{C}=\mathrm{C}), 1587(\mathrm{C}=\mathrm{N})$ & $5.92\left(\mathrm{~s}, 2 \mathrm{H}, \mathrm{OCH}_{2}\right), 6.70-7.62(\mathrm{~m}, 18 \mathrm{H}, \mathrm{Ar}-\mathrm{H})$ \\
\hline $4 \mathbf{f}$ & $\mathrm{C}_{29} \mathrm{H}_{22} \mathrm{~N}_{2} \mathrm{O}_{2}$ & $\begin{array}{c}80.98 \\
(80.93)\end{array}$ & $\begin{array}{c}5.17 \\
(5.11)\end{array}$ & $\begin{array}{c}6.56 \\
(6.51)\end{array}$ & $1611(\mathrm{C}=\mathrm{C}), 1587(\mathrm{C}=\mathrm{N})$ & $\begin{array}{l}5.12\left(\mathrm{~s}, 2 \mathrm{H},-\mathrm{CH}_{2}-\right), 6.05\left(\mathrm{~s}, 2 \mathrm{H}, \mathrm{OCH}_{2}\right), 6.69-7.60 \\
(\mathrm{~m}, 18 \mathrm{H}, \mathrm{Ar}-\mathrm{H})\end{array}$ \\
\hline $4 g$ & $\mathrm{C}_{30} \mathrm{H}_{20} \mathrm{CIN}_{3}$ & $\begin{array}{c}78.62 \\
(78.68)\end{array}$ & $\begin{array}{c}4.32 \\
(4.37)\end{array}$ & $\begin{array}{c}9.12 \\
(9.18)\end{array}$ & $1614(\mathrm{C}=\mathrm{C}), 1578(\mathrm{C}=\mathrm{N})$ & 7.21-8.32 (m, 20H, Ar-H + Quinolyl) \\
\hline $4 h$ & $\mathrm{C}_{31} \mathrm{H}_{22} \mathrm{ClN}_{3}$ & $\begin{array}{c}78.83 \\
(78.89)\end{array}$ & $\begin{array}{c}4.58 \\
(4.66)\end{array}$ & $\begin{array}{c}8.96 \\
(8.90)\end{array}$ & $1619(\mathrm{C}=\mathrm{C}), 1583(\mathrm{C}=\mathrm{N})$ & $5.1\left(\mathrm{~s}, 2 \mathrm{H},-\mathrm{CH}_{2}\right), 7.20-8.35(\mathrm{~m}, 19 \mathrm{H}, \mathrm{Ar}-\mathrm{H})$ \\
\hline
\end{tabular}


the structure of the product. The appearance of band at 1585 and $1600-1625 \mathrm{~cm}^{-1}$ due to $\mathrm{C}=\mathrm{N}$ and $\mathrm{C}=\mathrm{C}$ respectively further confirmed the formation of the products.

\section{Experimental Section}

Microwave irradiations were carried out in Kenstar Microwave Oven, Model No. OM9925E (2450 MHz, $800 \mathrm{~W}$ ) and IR spectra were recorded on a Perkin Elmer FTIR-1710 spectrophotometer using $\mathrm{KBr}$ pellets. ${ }^{1} \mathrm{H}$ NMR spectra were recorded on Bruker Avance 300 Spectrospin $(300 \mathrm{MHz}$ ) instrument. Temperature of the reaction mixture was measured through AZ, Mini Gun Type, Non Contact IR thermometer, Model No. 8868. The melting points were determined on a Thomas Hoover melting points apparatus and are uncorrected. Chemical shifts, $\delta$, for ${ }^{1} \mathrm{H}$ NMR, are given in ppm, relative to internal reference, tetramethylsilane (TMS) and IR frequency, $v$ (KBr pellets), in $\mathrm{cm}^{-1}$. The purity of compounds was checked on aluminium plates coated with silica gel (Merck).

General procedure for the synthesis of 2-substituted 4,5-diphenyl-1H-imidazole derivatives (2a-h). A mixture of $0.02 \mathrm{~mol}$ of benzil, $0.02 \mathrm{~mol}$ of aldehyde $\mathbf{1 a - h}$ and 0.07 mol of ammonium acetate were taken in an Erlenmeyer flask and was subjected to microwave irradiation. On completion of reaction as monitored by TLC at an interval of 30 seconds the sticky solid obtained was triturated with few drops of methanol to obtain the desired product $\mathbf{2 a - h}$ which was recrystallized from aqueous methanol.

General procedure for the synthesis of 1-phenyl/benzyl-2-substituted-4,5-diphenyl-1H-imidazole derivatives 4a-h. A mixture of $0.02 \mathrm{~mol}$ of benzil, $0.02 \mathrm{~mol}$ of aldehyde 1a-d, $0.02 \mathrm{~mol}$ of aromatic amine 3a-b and $0.07 \mathrm{~mol}$ of ammonium acetate were taken in an Erlenmeyer flask and was subjected to microwave irradiation. On completion of reaction as monitored by TLC at an interval of 30 seconds the sticky product obtained was triturated with few drops of appropriate solvent (methanol for $\mathbf{4 a}, \mathbf{b}, \mathbf{c}, \mathbf{f}$ and mixture of benzene: petroleum ether for $\mathbf{4 d}, \mathbf{e}, \mathbf{g}, \mathbf{h})$ to obtain the required product $\mathbf{4 a - h}$.

\section{Conclusion}

An highly efficient microwave assisted rapid synthesis of tri/ tetrasubstituted $1 \mathrm{H}$ imidazoles has been developed. This solvent free neat reaction is not only of interest from an ecological viewpoint but also offer considerable synthetic advantages in terms of yield, selectivity and simplicity of the reaction procedure. Though these solventless reactions are more appropriate for small scale productions but these results may stimulate interest among researchers involved in industrial scale up of reactions.

Acknowledgement. The authors $\mathrm{M}$. Kidwai and S. Saxena thanks University Grants Commission, New Delhi for the financial assistance.

\section{References}

1. Gedye, R.; Smith, F.; Westway, K.; Ali, H.; Baldisera, L.; Laberge, L.; Rousell, J. Tetrahedron Lett. 1986, 27, 279.

2. Varma, R. S.; Dahiya, R.; Kumar, S. Tetrahedron Lett. 1997, 38 , 2039.

3. (a) Caddick, S. Tetrahedron 1995, 51, 10403. (b) Bose, A. K.; Banik, B. K.; Lavlinskaia, N.; Jayaraman, M.; Manhas, M. S. Chemtech 1997, 27, 18

4. Clark, J. H. In Catalysis of Organic Reactions by Supported Inorganic Reagents; VCH Publisher, Inc: New York, 1984.

5. Varma, R. S. In Microwaves in Organic Synthesis; Loupy, A., Ed.; Wiley VCH: Weinheim, 2002; Chapter 6, pp 181-218.

6. Varma, R. S. In Microwaves Theory, Application in Material Processing IV; Clark, D. E., Sutton, W. H., Lewis, D. A., Eds.; American Ceramic Society: Westerville, Ohio, 1997; pp 357365.

7. Ahluwalia, V. K.; Kidwai, M. In New Trends in Green Chemistry; Anamaya Publishers: New Delhi, 2003.

8. Dittmer, D. C. Chem. Ind. 1997, 779.

9. Ganellin, C. R. In Medicinal Chemistry The Role of Organic Chemistry in Drug Research; Roberts, S. M.; Price, B. J., Eds.; Academic Press: New York, USA, 1985; pp 93-119.

10. Rotstein, D. M.; Kertesz, D. J.; Walker, K. A. M.; Swinney, D. C. J. Med. Chem. 1992, 35, 2818.

11. Sensui, H.; Ichikawa, J.; Sato, S. Jpn. Kokai Tokyo Koho JP 62,94,841; Chem. Abstr. 1987, 107, 187436q.

12. Liebl, R.; Handte, R.; Mildenberger, H.; Bauer, K.; Bieringer, H. Ger. Offen DE 3,604,042; Chem. Abstr. 1987, 108, 6018g.

13. (a) Ucucu, U.; Karaburun, N. G.; Isikdag, I. Farmaco 2001, 56, 285. (b) Wolkenberg, S. E.; Wisnosk, D. D.; Leister, W. H.; Wang, Y.; Zhao, Z.; Lindsley, C. W. Org. Lett. 2004, 6(9), 1453.

14. Pozherskii, A. F.; Soldatenkov, A. T.; Katritzky, A. R. Heterocycles in Life, Society; Wiley: New York, 1997; p 179.

15. Lombardino, J. G.; Wiseman, E. H. J. Med. Chem. 1974, 17, 1182.

16. Phillips, A. R.; White, H. L.; Rosen, S. Eur. Pat. Appl. EP 58,890; Chem. Abstr. 1982, 98, 53894z.

17. Radziszewski, B. Ber. 1882, 15, 1493.

18. Grimmett, M. R. In Comprehensive Heterocyclic Chemistry II; Katritzky, A. R.; Rees, C. W.; Scriven, E. F. V., Eds.; Pergamon: New York, 1996; Vol. 3, p 77.

19. (a) Balalaie, S.; Arabanian, A.; Hashtroudi, M. S. Montash. Chem. 2000, 131, 945. (b) Usyatinsky, A. Y.; Khmelnitsky, Y. L. Tetrahedron Lett. 2000, 41, 5031.

20. (a) Balalaie, S.; Hashem, M. M.; Akhbari, M. Tetrahedron Lett. 2003, 44(8), 1709. (b) Sarshar, S.; Siev, D.; Mjalli, M. M. Tetrahedron Lett. 1996, 37, 835.

21. Wasserman, H. H.; Long, Y. O.; Zhang, R.; Parr, J. Tetrahedron Lett. 2002, 43, 3351.

22. Kamitori, Y. J. Heterocycl. Chem. 2001, 38, 773.

23. (a) Kidwai, M.; Saxena, S.; Mohan, R.; Venkataramanan, R. J. C. S. Perkin Trans. 1 2002, 16, 1845. (b) Kidwai, M.; Mohan, R. Canadian J. Chem. 2004, 82, 427.

24. Krieg, B.; Manecke, G. Z. Naturforschg 1967, 22b, 132.

25. (a) Kidwai, M.; Rastogi, S.; Saxena, S. Bull. Korean Chem. Soc. 2004, 25(1), 119. (b) Kidwai, M.; Mothsra, P.; Mohan, R.; Biswas, S. Bioorg. Med. Chem. Lett. 2005, 15(14), 915.

26. (a) Davidson, D.; Weiss, M.; Jelling, M. J. Org. Chem. 1937, 2, 319; (b) Whiten, D. M.; Sonnenberg, J. J. Org. Chem. 1964, 29, 1926.

27. (a) Japp, F. R.; Robinson, H. H. Chem. Ber. 1882, 15, 1269. (b) Weiss, M. J. Org. Chem. 1952, 74, 5193.

28. Gompper, R. Chem. Ber. 1957, 90, 37. 